\title{
INVESTIGACIONES
}

\section{Beneficios de los programas intergeneracionales en el desarrollo de competencias emocionales}

\author{
Benefits of intergenerational programs \\ in the development of emotional competencies \\ Patricia Torrijos Fincias ${ }^{a}$, Sara Serrate González ${ }^{a}$ \\ ${ }^{a}$ Universidad de Salamanca. \\ patrizamora@usal.es,sarasg@usal.es
}

\begin{abstract}
RESUMEN
Desde la importancia del desarrollo de competencias socioemocionales como elemento clave para favorecer el bienestar personal y social, se diseña e implementa un programa intergeneracional de Educación Emocional, teniendo como objetivo evaluar la mejora de la competencia emocional de los participantes, a través de una investigación evaluativa de carácter mixto, bajo un diseño pre-experimental con medida pre-postest. La muestra participante son estudiantes de últimos cursos de Grado y Máster y estudiantes del Programa Universitario para Mayores de la Universidad de Salamanca. Las variables de estudio son: el interés hacia la formación y el nivel de desarrollo emocional autoinformado. Los resultados evidencian cambios en la autopercepción del nivel competencial a partir de la participación en el programa en ambos grupos, pudiéndose inferir que la formación les ha servido para aumentar su percepción en la dimensión competencias para la vida y bienestar.
\end{abstract}

Palabras clave: educación emocional, formación del profesorado, educación de adultos, intergeneracional, investigación evaluativa.

\begin{abstract}
From the importance of the development of social-emotional competences as a key element to favour the personal and social well-being, an intergenerational programme of Emotional Education is designed and implemented, having as an objective to evaluate the improvement of the emotional competence of the participants, through an evaluative research of mixed character, under a pre-experimental design with pre-post test measure. The participating sample are students in their final year of their Bachelor's and Master's degrees and students from the University Programme for the Elderly at the University of Salamanca. The study variables are: interest in training and level of self-reported emotional development. The results show changes in the self-perception of the level of competence from the participation in the program in both groups, being able to infer that the training has served them to increase their perception in the dimension competences for life and wellbeing.
\end{abstract}

Key words: emotional education, teacher training, adult education, intergenerational, evaluative research. 


\section{INTRODUCCIÓN}

El interés que se le concede al desarrollo de competencias socioemocionales como elemento clave para favorecer el bienestar personal y social se ha hecho innegable en los últimos años. Hacer efectiva una educación integral y de calidad exige empezar a conceder la importancia que se merece al desarrollo emocional en todas las etapas de la vida, como complemento idóneo al desarrollo cognitivo, no dejando estos aspectos relegados a la etapa escolar o al ámbito privado.

Dar respuesta a las demandas y necesidades sociales exige empoderar a los profesionales de la educación y promover toda una serie de competencias clave para atender a los desafíos de su práctica, así como establecer iniciativas educativas que favorezcan el reencuentro intergeneracional, promoviendo toda una serie de actitudes y valores que no siempre son atendidas en el currículo ordinario.

Tomando como punto de partida los beneficios y experiencia en educación emocional e innovando en una estrategia formativa que fomente el aprendizaje y el encuentro intergeneracional se fundamenta a continuación, bajo el diseño de una investigación evaluativa, la implementación y evaluación de los efectos de un programa de educación emocional, tomando en consideración el interés hacia la formación y el nivel de desarrollo emocional autoinformado de los participantes.

\section{SENTIDO DE LA EDUCACIÓN EMOCIONAL EN LA FORMACIÓN DE LOS PROFESIONALES DEL MUNDO EDUCATIVO}

La labor educativa de nuestro siglo asiste a innumerables procesos de transformación que van más allá de centrarnos en el desarrollo cognitivo (Delors, 1996; Imbernón, 2007). Problemas como la violencia en las aulas, el fracaso escolar, el consumo y abuso de sustancias o los altos índices de ansiedad y depresión, exigen el planteamiento de respuestas innovadoras que permitan hacer de los centros educativos verdaderos espacios de encuentro y de desarrollo, donde la tarea de los distintos agentes trascienda de la mera transmisión de conocimientos a la promoción y desarrollo de competencias y habilidades básicas para la vida, dando sentido a una verdadera educación integral y de calidad (Bisquerra Alzina, Pérez González y García Navarro, 2015; Brackett, Mayer y Warner, 2004, Conde y Muñoz, 2002; Feixas, 1999; Fernández-Berrocal y Ruíz-Aranda, 2008; Pacheco, Villagrán y Guzmán, 2015).

Reconociendo que la finalidad de la educación no es otra que el desarrollo humano, la educación emocional se convierte en una estrategia de prevención para disminuir los efectos de aquellos factores que pueden dificultar no solo el desarrollo, sino el bienestar personal y social de las personas, respondiendo a toda una serie de necesidades sociales (Bisquerra, 2003, 2013). Hablamos así de una forma de prevención primaria inespecífica, pues minimiza la vulnerabilidad a disfunciones y previene su ocurrencia, garantizando el afrontamiento ante las circunstancias de la vida con mayor garantía de éxito.

En los últimos años se han puesto en marcha multitud de iniciativas para promover competencias emocionales en los centros educativos (Collaborative for Academic Social and Emotional Learning, 2003; Informe de la Fundación Marcelino Botín, 2008; Petrides, Frederickson y Furnham, 2004) y, aun verificando los beneficios de los mismos, 
encontramos que la gran mayoría de estas propuestas están dirigidas al alumno como el principal beneficiario de la intervención, siendo escasas las investigaciones que centran su interés en la promoción de competencias emocionales del profesorado.

Trabajar por el desarrollo de competencias intra e interpersonales en los futuros docentes y responsables del proceso educativo, tales como la conciencia emocional, la regulación emocional o la empatía favorecerá habilidades de resolución de conflictos e influirá en la mejora de la convivencia en los centros. Se señala la necesidad de dar cabida a la educación emocional de los agentes educativos como paso previo y fundamental para incidir en el desarrollo de competencias emocionales de los beneficiarios directos (Collaborative for Academic Social and Emotional Learning, 2003; Saarni, 2000; Petrides, Frederickson y Furnham, 2004, Saavedra y Reynaldos, 2006; Veloso, Cuadra, Antezana, Avendaño y Fuentes, 2013; Zeidner, Mattews y Roberts, 2009).

Se trata de empoderar a los futuros profesionales en toda una serie de competencias socioemocionales que les permitirán hacer frente a los desafíos de su práctica (Romero y Pereira, 2011; Urrea, Rodríguez, Sala y Zárate, 2021), competencias que si bien se reflejan como transversales no se trabajan específicamente en cada una de las materias del currículum formativo de los profesionales.

\section{LA EDUCACIÓN EMOCIONAL EN LOS PROGRAMAS UNIVERSITARIOS PARA MAYORES}

Cuando se hace alusión a los Programas Universitarios para Mayores se hace referencia a programas de formación científica, cultural, tecnológica y social, diseñados y ejecutados por las distintas universidades, cuya formación se constituye como un medio eficaz para fomentar el envejecimiento activo, la mejora de la calidad de vida y la prevención de la dependencia (Pérez, 2012). Entre sus objetivos didácticos se destaca el fomento de la autonomía y la lucha contra el riesgo de exclusión social, al ofrecer actividades intelectuales, fomentar las relaciones interpersonales y permitir una mejor proyección social de la persona mayor. Son, en definitiva, un espacio de aprendizaje a lo largo de la vida que permiten a los mayores adquirir nuevos conocimientos, mejorar sus habilidades y competencias y promover el desarrollo de capacidades cognitivas, físicas y emocionales, ofreciendo así una educación integral, en la que no se priorice únicamente el desarrollo cognitivo, sino el desarrollo afectivo y emocional (Ortiz-Colón, 2015).

A tenor de las casi cuatro décadas desde el inicio de los Programas Universitarios para Mayores en España y de los resultados de las investigaciones desarrolladas en este línea (Compton, Bachman, Brand y Avet, 2000; Montoro y Pinzano, 2005; Muñoz, 2007; Plaza, Requena, Rosario y López, 2015; Wight, Aneshansel y Seeman, 2002), no se pone en duda que este tipo de iniciativas, en primer lugar, ayudan a los mayores a mantenerse activos mental y físicamente, propiciando una estimulación cognitiva y proporcionando efectos protectores o estrategias compensatorias que ayudan a mitigar declives cognitivos en edades avanzadas. En segundo lugar, contribuyen a la realización personal y les permite estar vinculados a la vida social, ayudando al establecimiento de nuevas redes de relación interpersonal, favoreciendo, de esta forma, la integración social de este colectivo (Dench y Reagan, 2000; Gázquez, Pérez, Fernández, González, Ruiz, y Díaz, 2009; Gázquez, Pérez-Fuentes y Carrión, 2010; Purdie y Bulton-Lewis, 2003). Y, en tercer lugar, este tipo 
de programas mejoran el bienestar al influir en el desarrollo de estrategias efectivas de afrontamiento ante las experiencias vitales estresantes (Jarvis, 2001; Manheimer, MoskowMckenzie y Snodgrass, 1995). Se ha demostrado igualmente que a medida que aumentan los años de participación en este tipo de programas aumentan las habilidades para resolver conflictos, los sujetos aprenden más de sí mismos, aceptan mejor a los otros y mejoran sus capacidades comunicativas (Montoro y Pinzano, 2005; Pérez-González, 2012).

De la misma manera, en estudios más específicos, se defiende que la participación en los Programas Universitarios para Mayores ejerce un efecto beneficioso sobre la memoria, la fluidez del vocabulario y reduce el declive mental asociado al envejecimiento (Vilaplana, 2016). Contar con metas que cumplir y percibir el aprendizaje como una recompensa personal también tiene un efecto positivo sobre el nivel de satisfacción (Vilaplana, 2010). En la misma línea, Ortiz-Colón (2015) asegura que dichos programas constituyen una estrategia idónea al favorecer la autoestima y motivación de los mayores al hacerles sentir útiles y válidos, capaces de aprender y participar activamente, lo que permite también un aumento de la satisfacción con la vida y una mejora la percepción del interés generativo (Navarro, Serrate, Muñoz y Díaz-Orueta, 2018).

Los Programas Universitarios para Mayores son, por tanto, propuestas que fomentan una vejez activa, interfiriendo de un modo positivo en la forma de hacerse mayor y en la manera de optimizar la conducta personal y social que deberían favorecer el bienestar emocional. No obstante, aunque expertos a nivel nacional e internacional defienden que la educación emocional debe estar presente a lo largo de la vida, son escasos los trabajos que centran su atención en la promoción de habilidades socioemocionales en adultos o mayores (Bisquerra, Pérez- González y García, 2015; Fernández-Berrocal y Extremera, 2006; Schulze y Roberts, 2005; Serrate, Torrijos y Navarro, 2018). Así, la mayor parte de los trabajos de educación emocional están dirigidos principalmente a niños y jóvenes dentro del contexto escolar, centrando su interés en el impacto que este tipo de programas tienen en el bienestar personal y social (Durlak, Domitrovich, Weissberg y Gullota, 2015; Pena, Extremera y Rey, 2016; Schutte, Malouff y Thorteinson, 2013).

\section{METODOLOGÍA}

El objetivo general de la investigación se centra en evaluar la mejora de la competencia emocional tras la aplicación de un programa intergeneracional de Educación Emocional, diseñado e implementado con estudiantes universitarios de los últimos cursos de Grado y Máster y estudiantes matriculados en Programas Universitarios para mayores. La evaluación de los efectos del programa sobre el nivel de competencias emocional autoinformado se ampara en el diseño de estudio pre-experimental con medida pre-test y pos-test (Campbell y Standley, 1963).

\subsection{MUESTRA}

La muestra del presente estudio estuvo compuesta por un total de 30 estudiantes, de los cuales, 15 fueron estudiantes provenientes de los últimos cursos de las titulaciones de Grado en Educación Social y Pedagogía, así como del Máster en Profesorado de Educación Secundaria Obligatoria, Formación Profesional y Enseñanzas Artísticas y de Idiomas y 15 
eran estudiantes del Programa Interuniversitario de la Experiencia de la Universidad de Salamanca. El número reducido de participantes en esta fase del estudio se debe al carácter metodológico de la formación implementada, que pretendía, no solo inculcar conocimiento en materia de Educación Emocional, sino facilitar experiencias para promover la competencia emocional de los participantes, trabajando comportamientos y actitudes que fomentaran el bienestar personal y social a través del encuentro intergeneracional.

Tabla 1. Características de la muestra participante

\begin{tabular}{|c|c|c|c|c|c|c|c|c|c|c|c|}
\hline \multicolumn{9}{|c|}{ JÓVENES } & \multicolumn{7}{c|}{ MAYORES } \\
\hline \multicolumn{2}{|c|}{ Hombres } & \multicolumn{2}{|c|}{ Mujeres } & \multicolumn{2}{|c|}{ Formación previa } & \multicolumn{2}{c|}{ Hombres } & \multicolumn{2}{c|}{ Mujeres } & Formación previa \\
\hline $\mathrm{f}$ & $\%$ & $\mathrm{f}$ & $\%$ & $\mathrm{f}$ & $\%$ & $\mathrm{f}$ & $\%$ & $\mathrm{f}$ & $\%$ & $\mathrm{f}$ & $\%$ \\
\hline 1 & 6,7 & 14 & 93,3 & 15 & 100 & 5 & 30,8 & 10 & 69,2 & 4 & 26,7 \\
\hline
\end{tabular}

El acceso a la formación fue de carácter voluntario (llevándose a cabo un proceso de selección sobre las 40 personas interesadas de acuerdo al orden de matriculación en el curso), contando finalmente con 24 mujeres y 6 hombres (diferencia de género que queda explicada de acuerdo a la mayor proporción de mujeres que participan en este tipo de programas). En cuanto a las edades oscilaban entre los 20 y 25 ( $M d=22,20 ; S x=1,26)$ en el caso de los jóvenes y los 55 y 68 años de edad $(\mathrm{Md}=63,23 ; \mathrm{Sx}=3,74)$ en el caso de los adultos. Al preguntarles por la formación previa, los universitarios más jóvenes informan de mayores conocimientos iniciales, reconociendo ser sus principales fuentes de información el haber trabajado estos temas en alguna asignatura o, en menor medida, la asistencia a otros cursos. En el caso de los mayores encontramos que solamente cuatro personas reconocen tener conocimientos previos, siendo sus principales fuentes formativas la asistencia a otros cursos y el haber leído libros sobre el tema.

\subsection{INSTRUMENTOS DE MEDIDA}

El instrumento utilizado para evaluar los cambios en la autopercepción de la competencia emocional fue el Cuestionario de Desarrollo Emocional para adultos CDE-A35, una versión reducida del CDE-A [Pérez-Escoda y Alegre, en prensa; Pérez-Escoda, Bisquerra, Filella y Soldevila (2010)]. El CDE-A es una medida de autoinforme utilizada para medir el nivel de competencia emocional autopercibido en la edad adulta. Está compuesto por 35 ítems en una escala tipo Likert de 11 puntos, que se puntúan de 0 a 10 en función del grado de acuerdo con cada una de las afirmaciones, donde el cero indica un total desacuerdo y el diez el acuerdo total. Se fundamenta en el marco teórico de la Educación Emocional desarrollado por el Grupo de Investigación GROP (Bisquerra, 2000; Bisquerra y Pérez Escoda, 2007), de acuerdo al modelo pentagonal que defiende que la competencia emocional está compuesta por cinco dimensiones: conciencia emocional (8 ítems), regulación emocional (8 ítems), autonomía emocional (5 ítems), competencias sociales (6 ítems) y competencias para la vida y el bienestar (8 ítems). 
Para la interpretación de las puntuaciones del cuestionario se trabajó con las puntuaciones promedio que corresponden a seis dimensiones, cinco adecuadas a cada una de las competencias del modelo pentagonal del GROP (Bisquerra y Pérez Escoda, 2007) y una dimensión adecuada al total de la prueba. Las puntuaciones oscilan entre 0 y 10 , siendo 0 carencia absoluta de competencia en la dimensión y 10 dominio absoluto.

La información aportada por dicho cuestionario fue complementada con un instrumento de carácter más cualitativo, a través de unos diarios finales de seguimiento que fueron realizando los participantes al finalizar cada uno de los encuentros formativos.

\subsection{PROCEDIMIENTO}

El programa Intergeneracional de Educación Emocional se implementó como un curso de carácter eminentemente práctico a lo largo de seis sesiones semanales de dos horas y media de duración cada una. La metodología didáctica del mismo se desarrolló través de la participación activa, fomentando oportunidades formativas con el propósito de trabajar la reflexión y la conciencia sobre cómo las emociones influyen en la adopción de determinadas conductas que no siempre son efectivas (Torrijos, 2016). A partir del reconocimiento de estos comportamientos, se promovió el cambio de acciones, pensamientos, actitudes y emociones, buscando mayor efectividad y haciéndoles partícipes de la búsqueda del propio equilibrio emocional. Por lo tanto, se trató de favorecer el aprendizaje de las competencias emocionales a partir de la experiencia personal, partiendo de la diversidad y del constante intercambio intergeneracional. La metodología utilizada fue, por lo tanto, activa y participativa, poniendo en práctica actividades del tipo: a) regulación, análisis y reconstrucción de situaciones vividas, b) juegos de roles e interpretación, c) juegos cooperativos, d) debates, e) análisis de casos sobre texto y vídeo. Los contenidos específicos trabajados a lo largo del programa quedan recogidos en la tabla 2.

Tabla 2. Contenidos trabajados en el programa de Educación Emocional

\begin{tabular}{|l|l|}
\hline Sesión & Contenidos \\
\hline Sesión I & La inteligencia emocional, educación emocional y competencia \\
\hline Sesión II & Competencias emocionales y bienestar \\
\hline Sesión III & El papel cultural de las emociones: claves para su reconocimiento personal y social \\
\hline Sesión IV & Autorregulación y gestión emocional \\
\hline Sesión V & Automotivación, logro y éxito personal \\
\hline Sesión VI & Comunicación afectiva y efectiva: empatía y asertividad \\
\hline
\end{tabular}

La estrategia evaluativa del programa consistió en implementar la evaluación inicial para comprobar las necesidades previas autoinformadas y poder adaptar la propuesta formativa a las necesidades de los participantes y para ello se aplicó el CDE-A35 en la primera sesión, antes de comenzar con el desarrollo de los contenidos y la puesta en 
práctica de las actividades en las diferentes sesiones. Finalmente, para profundizar en la incidencia de la formación en cuanto a la autopercepción de la competencia emocional de los participantes se llevó a cabo una nueva aplicación en la última sesión (evaluación final), una vez que habían finalizado la formación los estudiantes.

Del mismo modo, se complementó dicha información con otros instrumentos de corte cualitativo, a partir de los diarios de sesiones que fueron realizando los participantes al finalizar cada uno de los encuentros formativos. Se utilizó, por tanto, una técnica de evaluación no estructurada y de carácter formativo. Se trata de un diario de tipo reflexivo (reflective writing) e introspectivo (intreospective wrtiting), en el que se pide a los participantes que reflexionen en torno al aprendizaje de cada una de las sesiones, es decir, en torno a los pensamientos, sentimientos o vivencias que experimentan a lo largo del proceso formativo. Entre las razones que nos llevan a incorporar el diario de seguimiento a nuestro estudio destacamos, principalmente, su utilidad como instrumento de investigación y desarrollo personal, donde el carácter abierto de esta herramienta permite una mayor riqueza informativa que va más allá de la mera descripción de los contenidos trabajados, proporcionándole al participante la oportunidad de ser agente activo en el proceso formativo. Además, posibilita realizar una lectura diacrónica de distintos acontecimientos que no quedan recogidos mediante el uso de otros instrumentos (Hernández Sampieri, Fernández Collado y Baptista, 2010; Colás Bravo, 1994; Tojar, 2006; Zabalza, 2004).

Estos seguimientos son recogidos por los dinamizadores del programa, ofreciendo una constante información sobre el proceso formativo. Finalizada la formación y una vez recogidas las aportaciones de los participantes, se lleva a cabo el análisis cualitativo de esta información, a través de la técnica de análisis de contenido (Rodríguez-Gómez, GilFlores y García-Jiménez, 1996), codificando cada uno de los seguimientos y estableciendo categorías que permiten analizar la información de forma conjunta, de acuerdo a una serie de tópicos, señalando en este artículo concretamente la satisfacción hacia el curso formativo.

El análisis de las respuestas ofrecidas por los participantes en la aplicación del CDE-A35 y el posterior tratamiento de los datos se realizó con el paquete estadístico SPSS. En un primer análisis descriptivo contamos con la puntuación directa de cada uno de los participantes en las distintas dimensiones, evaluando el potencial de desarrollo grupal en las cinco competencias. De este primer análisis descriptivo y exploratorio por variable se procede a depurar el archivo, conocer la distribución de las variables y, por lo tanto, determinar las técnicas inferenciales adecuadas para comprobar la existencia de diferencias significativas tomando como referencia la participación en el programa.

Para el estudio de las diferencia de puntuaciones en cada una de las dimensiones del CDE-A35, teniendo en cuenta el tamaño de la muestra así como el estudio del ajuste a la normalidad de la muestra, mediante las pruebas de Kolmogorov-Smirnov (con la corrección de Lilliefors) y Shapiro-Wilk, al no encontrarse evidencias suficientes para afirmar que las distribuciones de las variables objeto de estudio procedían de una distribución normal (p.<,05), se realizó un contraste no paramétrico para muestras relacionadas, aplicando el test de Wilcoxon, así como el tamaño del efecto de acuerdo a los criterios de correlación propuesta por Corder y Foreman (2009)1.

La fórmula para este cálculo es: $\mathrm{r}=[\mathrm{z}] / \sqrt{\mathrm{N}}$ 


\section{RESULTADOS}

\subsection{NIVEL DE COMPETENCIA EMOCIONAL AUTOINFORMADO}

El Cuestionario de Desarrollo Emocional inicial (pre-test) fue cumplimentado por un total de 28 participantes y 24 en la aplicación final (pos-test). Al igual que en la fase anterior, todos los participantes fueron informados del objeto de estudio, se les informó que la participación en el proceso evaluativo pre-postest tenía un carácter voluntario y se les aseguró del anonimato y confidencialidad de sus respuestas.

El análisis descriptivo de los resultados obtenidos en la aplicaciones pre-test del Cuestionario de Desarrollo Emocional para adultos CDE-A35 (véase tabla III), evidencia que los valores medios mínimos informados se registran en la dimensión de competencia social $(\mathrm{Md}=4,61 ; \mathrm{Sx}=1,46)$ y conciencia emocional $(\mathrm{Md}=5,62, \mathrm{Sx}=1,76)$ en el caso de los alumnos del grupo de jóvenes, mientras que en el caso de los mayores se evidencian en las dimensiones de autonomía social $(\mathrm{Md}=3,88, \mathrm{Sx}=1,47)$, obteniéndose puntuaciones medias inferiores en el caso del grupo de mayores en todas las dimensiones del instrumento. En la evaluación de la aplicación pos-test del cuestionario, se evidencian mayores puntuaciones medias en el grupo de mayores en la puntuación global y en 4 de las 5 dimensiones. De esta misma manera, en el grupo de jóvenes se registran también los resultados más bajos en las dimensiones de regulación emocional $(\mathrm{Md}=3,35, \mathrm{Sx}=1,17)$ y en competencia social $(\mathrm{Md}=3,30, \mathrm{Sx}=1,84)$, tanto en competencia social $(\mathrm{Md}=3,97, \mathrm{Sx}=2,28)$ como en regulación emocional $(\mathrm{Md}=3,94, \mathrm{Sx}=1,52)$.

Tabla 3. Valores Pre-test-Pos-test Cuestionario de Desarrollo Emocional para adultos CDE-A

\begin{tabular}{|c|c|c|c|c|c|c|c|}
\hline \multicolumn{2}{|c|}{ Global QDE-A } & \multirow{2}{*}{$\begin{array}{c}\text { Jóvenes } \\
6,74\end{array}$} & \multirow{2}{*}{$\begin{array}{c}\text { Mayores } \\
6,30\end{array}$} & \multicolumn{2}{|c|}{ Conciencia Emocional } & Jóvenes & Mayores \\
\hline \multirow{4}{*}{ Pre-test } & $\bar{X}$ & & & \multirow{4}{*}{ Pre-test } & $\bar{X}$ & 5,62 & 4,54 \\
\hline & $S_{x}$ & 0,96 & 1,51 & & $S_{x}$ & 1,76 & 1,87 \\
\hline & Mínimo & 5,50 & 3,88 & & Mínimo & 2,63 & 2,50 \\
\hline & Máximo & 8,50 & 9,75 & & Máximo & 8,25 & 8,50 \\
\hline \multirow{4}{*}{ Pre-test } & $\bar{X}$ & 5,02 & 5,87 & \multirow{4}{*}{ Pos-test } & $\bar{X}$ & 6,35 & 6,84 \\
\hline & $S_{x}$ & 0,57 & 1,04 & & $S_{x}$ & 0,51 & 0,90 \\
\hline & Mínimo & 4,19 & 4,34 & & Mínimo & 5,50 & 5,38 \\
\hline & Máximo & 5,93 & 8,06 & & Máximo & 7,38 & 8,75 \\
\hline \multicolumn{2}{|c|}{ Regulación emocional } & Jóvenes & Mayores & \multicolumn{2}{|c|}{ Competencia social } & Jóvenes & Mayores \\
\hline \multirow{4}{*}{ Pre-test } & $\bar{X}$ & 5,83 & 4,51 & \multirow{4}{*}{ Pre-test } & $\bar{X}$ & 4,61 & 4,51 \\
\hline & $S_{x}$ & 1,99 & 1,87 & & $S_{x}$ & 1,46 & 2,02 \\
\hline & Mínimo & 2,80 & 2,50 & & Mínimo & 3,00 & 1,40 \\
\hline & Máximo & 9,00 & 8,50 & & Máximo & 7,67 & 8,60 \\
\hline
\end{tabular}




\begin{tabular}{|c|c|c|c|c|c|c|c|}
\hline & $\bar{X}$ & 3,35 & 4,52 & \multirow{4}{*}{ Pos-test } & $\bar{X}$ & 3,30 & 4,63 \\
\hline \multirow{3}{*}{ Pos-test } & $S_{x}$ & 1,17 & 1,65 & & $S_{x}$ & 1,84 & 2,55 \\
\hline & Mínimo & 1,63 & 1,88 & & Mínimo & 1,20 & 0,8 \\
\hline & Máximo & 5,13 & 6,88 & & Máximo & 7,00 & 8,80 \\
\hline \multicolumn{2}{|c|}{ Autonomía Social } & Jóvenes & Mayores & \multicolumn{2}{|c|}{ Competencias vida y bienestar } & Jóvenes & Mayores \\
\hline \multirow{4}{*}{ Pre-test } & $\bar{X}$ & 7,22 & 3,88 & \multirow{4}{*}{ Pre-test } & $\bar{X}$ & 6,09 & 5,25 \\
\hline & Sx & 0,93 & 1,47 & & $S_{x}$ & 0,99 & 1,02 \\
\hline & Mínimo & 5,25 & 1,33 & & Mínimo & 4,49 & 4,00 \\
\hline & Máximo & 8,25 & 6,67 & & Máximo & 7,74 & 7,71 \\
\hline \multirow{4}{*}{ Pos-test } & $\bar{X}$ & 4,18 & 6,11 & \multirow{4}{*}{ Pos-test } & $\bar{X}$ & 7,92 & 7,20 \\
\hline & Sx & 1,60 & 1,51 & & $S_{x}$ & 0,78 & 1,42 \\
\hline & Mínimo & 1,17 & 3,68 & & Mínimo & 6,75 & 4,25 \\
\hline & Máximo & 6,33 & 9,50 & & Máximo & 8,88 & 8,75 \\
\hline
\end{tabular}

El estudio de comparación de las puntuaciones medias informadas de acuerdo a ambos grupos a los resultados pre-postest (véase figura 1) nos permite evidenciar una tendencia favorable al postest en ambos grupos en la dimensión de conciencia emocional y competencias para la vida y el bienestar, así como una tendencia desfavorable al postest en el caso de ambos grupos en la dimensión de regulación emocional, evidenciándose puntuaciones medias inferiores en las dimensiones de regulación, competencia social y autonomía emocional.

Figura 1. Comparación puntuaciones en CDE-A (Pre-test y Pos-test)

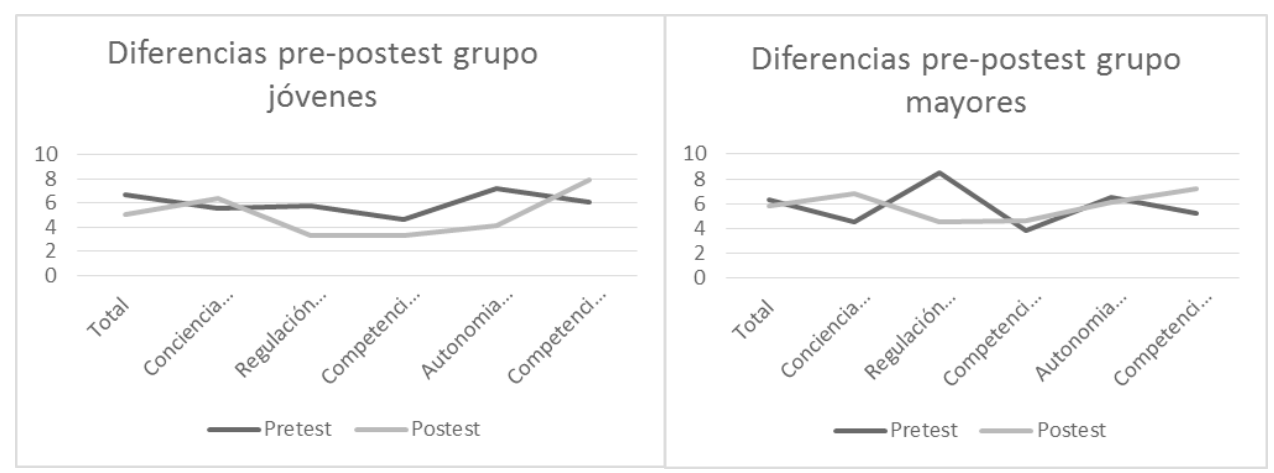


A partir de este primer análisis descriptivo, para conocer los efectos del programa sobre ambos grupos, se procede a profundizar en la diferencia de puntuaciones en cada una de las dimensiones del CDE-A35, de acuerdo al estudio para muestras relacionadas para aquellos que respondieron al cuestionario tanto en la fase pre como pos-test $(n=24)$. El estudio de diferencia de medias (tal como se muestra en la tabla 4) revela diferencias significativas en 4 de las 6 dimensiones evaluadas en el caso del grupo de jóvenes (alpha $<.05)$ : en la puntuación global, así como en las dimensiones de regulación emocional, autonomía emocional y competencias para la vida y bienestar. Del mismo modo, y dado el carácter de la muestra, se registran tamaños del efecto medios.

Tabla 4. Diferencia de medias en las dimensiones del CDE-A (Pre-test y Pos-test)

\begin{tabular}{|c|c|c|c|c|c|c|}
\hline & \multicolumn{3}{|c|}{ Estudiantes Jóvenes } & \multicolumn{3}{|c|}{ Estudiantes Mayores } \\
\hline & $\mathrm{Z}$ & $\mathrm{P}$ & $\mathrm{r}$ & $\mathrm{Z}$ & $\mathrm{p}$ & $\mathrm{r}$ \\
\hline $\begin{array}{l}\text { Puntuación global Pre-test -Puntuación global } \\
\text { Pos-test }\end{array}$ & $-2,981 b$ & 0,003 & 0,6 &,$- 235 b$ & 0,814 & 0,04 \\
\hline $\begin{array}{l}\text { Conciencia emocional Pre-test -Conciencia } \\
\text { emocional Pos-test }\end{array}$ & $-1,512 \mathrm{c}$ & 0,13 & 0,3 & $-2,317 \mathrm{c}$ & 0,021 & 0,47 \\
\hline $\begin{array}{l}\text { Regulación emocional Pre-test -Regulación } \\
\text { emocional Pos-test }\end{array}$ & $-1,883 b$ & 0,06 & 0,38 &, $000 \mathrm{~d}$ & 1 & 0 \\
\hline $\begin{array}{l}\text { Competencia social Pre-test -Competencia } \\
\text { social Pos-test }\end{array}$ & $-1,412 b$ & 0,158 & 0,29 &,$- 589 \mathrm{c}$ & 0,556 & 0,12 \\
\hline Autonomía Pre-test -Autonomía Pos-test & $-2,981 b$ & 0,003 & 0,61 &,$- 392 b$ & 0,695 & 0,08 \\
\hline $\begin{array}{l}\text { Competencia vida Pre-test -Competencia vida } \\
\text { Pos-test }\end{array}$ & $-2,981 \mathrm{c}$ & 0,003 & 0,61 & $-2,589 \mathrm{c}$ & 0,01 & 0,53 \\
\hline
\end{tabular}

En el caso de los adultos mayores las diferencias significativas se presentan en las dimensiones de conciencia emocional y competencias para la vida y bienestar, con tamaños del efecto medios.

\subsection{SATISFACCIÓN Y VALORACIÓN DE LA UTILIDAD DEL PROCESO FORMATIVO}

Para el análisis del contenido del discurso reflejado por los participantes en cada uno de los seguimientos se procede a la transcripción manual de la información. Dicha transcripción es realizada por varios investigadores evitando sesgos de interpretación y la pérdida de información. Para salvaguardar la identidad de los participantes, se le otorga a cada participante un código de identificación, así como el grupo (G1: Mayores, G2: Jóvenes) y el número de seguimiento, representado con una $\mathrm{S}$ acompañada del número del respectivo seguimiento (ej.1, 2, 3).

El proceso de codificación de la información se llevó a cabo mediante un sistema de categorías de carácter inductivo (Miles y Huberman, 1994), en el que participan 3 miembros del grupo de investigación Procesos, Espacios y Prácticas Educativas - GIPEP- de la 
Universidad de Salamanca con experiencia en este tipo de análisis, quedando estructuradas en 2 grandes tópicos: satisfacción y utilidad que aglutinan las categorías que se presentan en la tabla 5 .

Tabla 5. Tópicos y categorías de los análisis de contenido

\begin{tabular}{|l|l|}
\hline Tópicos & Categorías \\
\hline \multirow{4}{*}{ Satisfacción } & Desarrollo personal \\
\cline { 2 - 2 } & Aprendizajes \\
\cline { 2 - 2 } & Relaciones \\
\cline { 2 - 2 } & Metodología \\
\hline \multirow{4}{*}{ Utilidad } & Beneficios \\
\cline { 2 - 2 } & Aplicación práctica \\
\cline { 2 - 2 } & Necesidades autopercibidas \\
\cline { 2 - 2 } & Interés \\
\hline
\end{tabular}

Codificada la información en cada una de las categorías se procedió a comprobar en qué tópicos se centraban más los grupos participantes en cada uno de los seguimientos, información que queda recogida en la Figura 2. Se evidencia, en el caso de los jóvenes, un mayor peso en el tópico relacionado a la utilidad, especialmente, a medida que avanzan en el proceso formativo, valorando en mayor proporción la metodología práctica en las primeras sesiones.

En el caso de los mayores se refleja una mayor frecuencia de comentarios relacionados con la satisfacción personal que les produce la formación en aspectos relacionados con el desarrollo personal, los aprendizajes y las relaciones:

Este curso me parece muy interesante, por el intercambio con jóvenes que desde mi punto de vista es muy refrescante porque ellos tienen sabiduría y nosotros experiencia. A parte los jóvenes son maravillosos, preparados y la inmensa mayoría son muy solidarios. Es interesante y la profesora lo hace interesante, se nota que ha trabajado con jóvenes.

Participante 9_G1_S2_Estudiante UNEX 
Figura 2. Análisis del contenido en cada uno de los seguimientos.

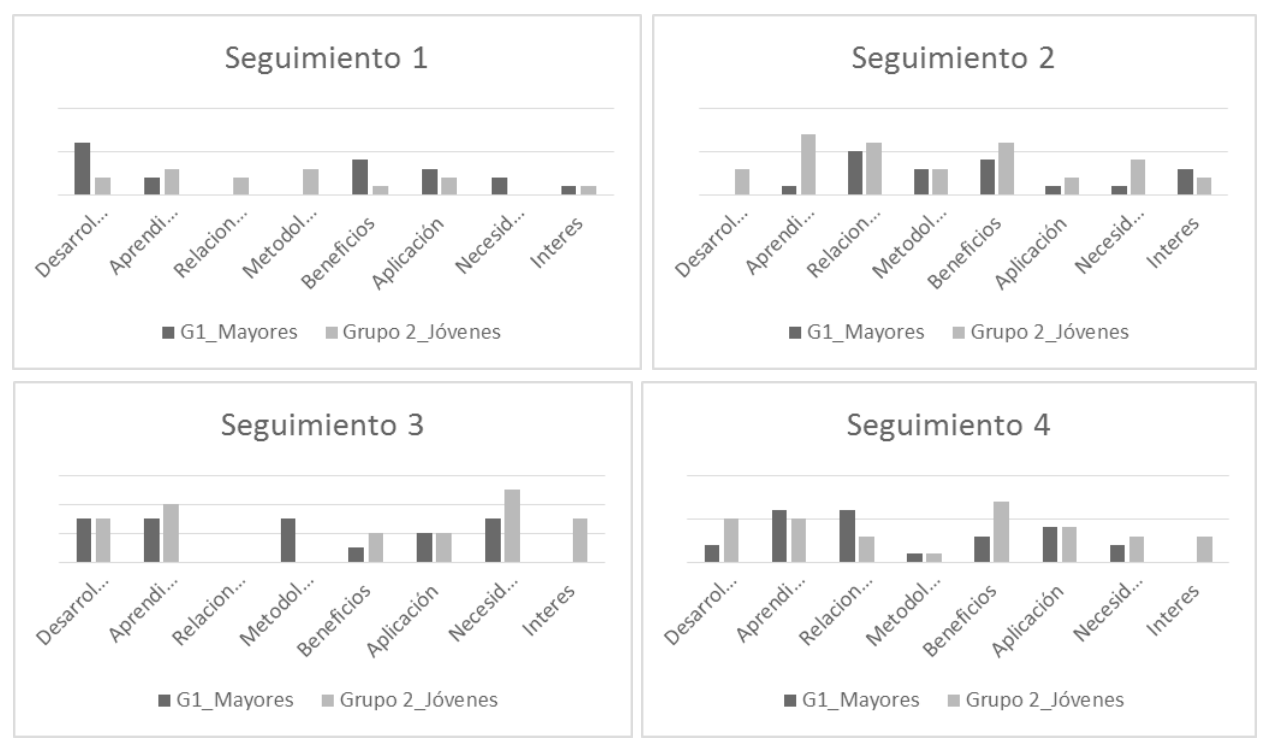

Entre los comentarios a los seguimientos, se evidencia nuevamente un mayor desconocimiento entre el grupo de mayores $(\mathrm{G} 1)$ :

Me ha resultado muy interesante, es una materia totalmente nueva para mí, creo que me servirá para conocerme mejor y para conocer mejor a los demás. Intuyo que me voy a encontrar en situaciones quizá comprometidas, pero soy consciente y responderé como se merece. Cada día es una aventura nueva para mí. Me ha gustado mucho la clase de hoy.

Participante 11_G2_S1_Estudiante UNEX

En el caso de los jóvenes encontramos que la dimensión de satisfacción guarda una gran relación con los aprendizajes y con los beneficios que perciben del proceso formativo:

Esta sesión ha sido interesante un reto ya que me cuesta mucho referirme a mí misma con calificativos positivos. Ha sido una gran experiencia y he podido lograr expresarme un poco más. También he aprendido el valor del trabajo en equipo para así poder regular la competitividad que se nos es impuesta.

Participante 16_G2_S3_Estudiante Pedagogía

Cuarta sesión: hoy he llegado realmente a emocionarme, esta sesión me ha hecho pensar en mí, pero también he pensado mucho en los míos. Me ha encantado lo que me han transmitido mis compañeros del curso, sentir que la gente piensa cosas tan bonitas de ti sin conocerte, son de verdad sensaciones increíbles.

Participante 27_G2_S4_Estudiante Máster 
Por otro lado, no sólo las relaciones interpersonales han sido un aspecto valorado muy positivamente, sino que a medida que participaban de la experiencia se reconocía un aumento del interés y de la necesidad de trabajar aspectos como la identificación de emociones o la regulación emocional:

La sensación que se me ha quedado unos días después de acabar el curso es ambigua. Siento que he avanzado en mi bienestar y creo que me ha impactado de una manera positiva, pero a la vez me ha dejado con el "gusanillo" de querer saber más.

Participante 18_G2_S4_Estudiante Educación Social

\section{DISCUSIÓN Y CONCLUSIONES}

Los resultados del estudio permiten afirmar cambios en la autopercepción del nivel competencial a partir de la participación en el programa en ambos grupos. Si bien existen diferencias en los niveles de autopercepción de la muestra participante, podemos inferir que en ambos grupos la participación en un curso formativo les ha servido para aumentar notablemente su percepción entorno a la capacidad de autodeterminación y resiliencia, relacionada con la dimensión que engloba competencias para la vida y bienestar, es decir, con todo ese conjunto de capacidades que favorecen la adopción de adoptar comportamientos responsables y orientados hacia la mejora del bienestar personal y social ante las demandas personales, profesionales y sociales (Bisquerra y Pérez-Escoda, 2007; Torrijos y PérezEscoda, 2014).

Si bien el lector puede considerar que en el postest se evidencian puntuaciones medias inferiores en cada una de las dimensiones, se debe tener en cuenta toda una serie de variables intervinientes propias de la investigación evaluativa como la deseabilidad social inicial, por ejemplo, así como la familiaridad con el investigador que puede inferir notablemente en las respuestas ofrecidas o la variabilidad de la muestra participante. Por otro lado, las diferencias en la dimensión de conciencia emocional en ambos grupos evidencia, una vez más, el impacto de la formación en educación emocional en el aumento de las necesidades autopercibidas cuando se participa en este tipo de experiencias formativas o, lo que es lo mismo, una vez se empieza a experimentar con algunas actividades, los participantes (en especial los que menos formación previa afirmaban tener) van siendo más conscientes de en qué aspectos o competencias deben incidir, algo que ha sido comprobado en estudios previos como los de Pérez González, Cejudo y Benito (2015) o Torrijos (2016).

Se muestra, una vez más, que el rol emocional de las personas, con especial incidencia en los adultos mayores, mejora tras la participación en un programa de educación emocional, mejorando con ello la percepción que el adulto tiene de su propio bienestar o sobre su autopercepción socioemocional (Pérez-Fuentes, Molero, Gázquez y Soler, 2014; Pérez-Fuentes, Gázquez, Molero, Martínea, Barragán y Simón, 2016; Soldevilla, Ribes, Filella y Aguilló, 2010). Unido a la idea de que la inteligencia emocional cambia a lo largo de la vida pudiendo incluso mejorar y que se trata de un constructo que se puede entrenar (Bar-On 1997; Chen, Peng y Fang, 2016; John y Gross, 2004; Kafetsios, 2004; Navarro, Latorre y Ros, 2009; Mayer, Caruso, Salovey, 2000; Sliter, Chen, Withrow y Sliter, 2013; Ugarriza, 2001) la investigación en este campo, parece estar demostrando que efectivamente los programas de educación emocional en la formación de profesionales 
y dentro de los programas universitarios para mayores están ofreciendo resultados satisfactorios. No obstante, se debe continuar evaluando el impacto que tiene este tipo de formación a lo largo del tiempo, para lo cual se plantea continuar con un estudio de carácter longitudinal, así como profundizar en el análisis de instrumentos más de corte cualitativo que se emplearon a lo largo del proceso formativo, a través de un estudio de casos, dado el carácter metodológico del proceso formativo.

Se convierte, por tanto, en un reto actual el incluir la educación emocional en el diseño y la programación de los distintos itinerarios de los Programas Universitarios para Mayores, así como en los itinerarios formativos de los futuros profesionales del mundo educativo, otorgándole "al saber ser y estar" el valor que le corresponde y más, si queremos seguir incidiendo en la educación integral y en la educación para y a lo largo de la vida (Delors, 1996; Navarro, Serrate, Muñoz y Díaz-Orueta, 2018). Y, en el diseño de los mencionados planes e itinerarios formativos, también apostamos por la implementación de experiencias intergeneracionales, puesto que, los resultados del presente estudio permiten afirmar que este tipo de metodología, específicamente para trabajar aspectos socioemocionales, ha tenido un impacto beneficioso y positivo para todos los implicados, al permitir un espacio de reflexión, debate y aprendizaje abierto y comprometido, aspectos ya reflejados en trabajos previos en esta misma línea (Amilburu, 2021; Gutiérrez y Hernández, 2013; Moreno, Martínez y Escarbajal, 2018; Ortega, 2021; Torrijos y Serrate, 2017).

Respecto a la aplicación del programa, conviene replicar el estudio ampliando la temporalización de este tipo de propuestas e incluso planteando distintos planteamientos metodológicos en la propuesta, así como poder establecer seguimiento de los participantes para poder comprobar la aplicación que los sujetos hacen de los conocimientos adquiridos y las competencias adquiridas a largo plazo.

No obstante, y a pesar de las limitaciones mencionadas, este trabajo es relevante en tanto que no existen estudios similares en la población adulta y mayor y en la formación de docentes, suponiendo un importante esfuerzo por esclarecer y ofrecer pequeños avances de carácter científico desde un enfoque metodológico e integrando una mayor riqueza a través de la experiencia de los mayores y de los deseos de los futuros profesionales por desarrollar toda una serie de competencias. De esta manera, se han obtenido evidencias que nos permiten continuar con la línea de investigación iniciada, en un intento de enfatizar la importancia de promover el desarrollo socioemocional, adaptándonos a las diferentes necesidades autopercibidas y a las características propias del ciclo vital de los participantes, suponiendo un importante paso para favorecer una adecuada educación integral y el desarrollo de competencias a partir del encuentro intergeneracional.

\section{REFERENCIAS BIBLIOGRÁFICAS}

Amilburu, M. G. (2021). Conexiones en red con otros tiempos, espacios y generaciones. Roger Scruton, tradición y educación. Teoría de la Educación. Revista Interuniversitaria, 33(1), 3549. DOI: $10.14201 /$ teri.23463

Bar-on, R. (1997). The Bar-On Emotional Quotient Inventory (EQ-i): A Test of Emotional Intelligence. Multi-Health Systems

Bisquerra, R. (2000). Educación emocional y bienestar. Praxis.

. (2003). Educación emocional y competencias básicas para la vida. Revista de Investigación Educativa, 21(1), 7-43. 
(2013). Cuestiones sobre bienestar. Síntesis.

Bisquerra, R. y Pérez-Escoda, N. (2007). Las competencias emocionales. Educación XXI, 10, 61-82. DOI: $10.5944 /$ educxx1.1.10.297

Bisquerra, R., Pérez-González, J. C. y García E. (2015). Inteligencia emocional en educación. Síntesis.

Brackett, M, Mayer, J. \& Warner, R. (2004). Emotional intelligence and its relation to everyday behavior. Personality and Individual Differences, 36, 1387-1402. DOI:10.1016/S01918869(03)00236-8.

Campbell, D. T. \& Standley J. C. (1963). Experimental and quasi-experimental designs for research. Handbook of Research on Teaching.

Chen, Y., Peng, Y. \& Fang, P. (2016). Emotional Intelligence Mediates the Relationship between Age and Subjective Well-Being. International Journal Aging Human Development, 83(2), 91-107. doi:10.1177/0091415016648705.

Colás, E. (1994). La investigación - acción. En E. Colás Bravo, y L. Buendía (Eds.), Investigación educativa (pp. 315-391). Alfar.

Collaborative for Academic, Social and Emotional Learning (2003). Safe and sound: An educational leader's guide to evidence-based social and emotional learning (SEL) programs. US Department of Education, Mid-Atlantic Regional Education Laboratory.

Compton, D. M., Bachman, L. D., Brand, D. y Avet, T. L. (2000). Age-associated changes in cognitive function in highly educated adults: Emerging myths and realities. International Journal of Geriatric Psychiatry, 15, 75-85. DOI: 10.1002/(sici)1099-1166(200001)15:1<75::aidgps81>3.0.co;2-m

Conde, H. \& Muñoz, J. M. (2002). Políticas comunitarias de juventud: el factor territorial en la sociedad global. Ediciones Universidad de Salamanca.

Corder, G. \& Foreman, D. (2009). Nonparametric statistics for non-statisticians: A stepby-step approach. Wiley.

Delors, J. (1996). La educación encierra un tesoro. Informe de la Comisión Internacional sobre la Educación para el siglo XXI. UNESCO-Santillana.

Dench, S. \& Regan, J. (2000). Learning in later life: Motivation and impact. DfEE.

Díaz, C. y García, N. (2016). Identificación de elementos relevantes para promover intervenciones eficaces en la vejez. Revista de psicodidáctica, 21, 157-173. DOI: 10.1387/RevPsicodidact.13854

Durlak, J. A., Domitrovich, C. E., Weissberg, R. P. \& Guilottta. T. P. (2015). Handbook of Social and Emotional Learning. Guilford Press.

Feixas, G. (1999). Prólogo. En M. Güell y J. Muñoz (Eds.), Desconócete a ti mismo. Programa de alfabetización emocional, (pp. 14-15). Paidós.

Fernández-Berrocal, P. y Extremera. N. (2006). La investigación de la inteligencia emocional en España. Ansiedad y Estrés, 12(3), 139-153. https://www.researchgate.net/publication/230886996_La_ investigacion_de_la_inteligencia_emocional_en_Espana

Fernández-Berrocal, P. y Ruiz-Aranda, D. (2008). La inteligencia emocional en la educación. Revista electrónica de investigación educativa, 15(6/2), 421-436.

Gázquez, J. J., Pérez, M. C., Fernández, M., González, L., Ruiz, I. \& Díaz, A. (2009). Old-age stereotypes related to the gerontology education: an intergenerational study. European Journal of Education and Psychology, 2(3), 263-273. DOI: 10.30552/ejep.v2i3.27

Gázquez, J. J., Pérez-Fuentes, M. C. y Carrión, J. J. (2010). Análisis de la memoria cotidiana en alumnos del Programa Universitario para Mayores. European Journal of Education and Psychology, 3(1), 155-165. DOI: 10.30552/ejep.v3i1.36

Gutiérrez, M. y Hernández, D. (2013). Los beneficios de los programas intergeneracionales desde la perspectiva de los profesionales. Pedagogía Social. Revista Interuniversitaria, (21), 213-235. https://dialnet.unirioja.es/servlet/articulo?codigo=4092197

Hernández-Sampieri, R., Fernández-Collado, C. y Baptista, M. P. (2010). Metodología de la investigación. Mc Graw Hill. 
Estudios Pedagógicos XLVII Nº 3: 273-290, 2021

BENEFICIOS DE LOS PROGRAMAS INTERGENERACIONALES EN EL DESARROLLO DE COMPETENCIAS

EMOCIONALES

Inbernom, F. (2007). 10 ideas clave: la formación permanente del profesorado: nuevas ideas para formar en la innovación y el cambio. Grao.

INE (2015). Índice de envejecimiento poblacional. Madrid: Instituto Nacional de Estadística.

Informe de la Fundación Marcelino Botín (IFBM, 2008). Educación Emocional y Bienestar. Análisis Internacional. Fundación Marcelino Botin.

Jarvis, P. (2001). Learning in later life: An introduction for educators and carers. Kogan Page.

John, O. P. \& Gross, J. J. (2004). Healthy and unhealthy emotion regulation: Personality processes, individual differences, and life span development. Journal of Personality, 72(6),1301-1333. DOI: 10.1111/j.1467-6494.2004.00298.x.

Kafetsios, K. (2004). Attachment and emotional intelligence abilities across the life course. Personality and Individual Differences, 37(1), 129-145. DOI: 10.1016/j.paid.2003.08.006.

Manheimer, R. J., Snodgrass, D. \& Moskow-Mckenzie, D. (1995). Older adults' education: A guide to research, policy and programs. Greenwood.

Mayer, J. D., Caruso, D. R. \& Salovey, P. (2000). Selecting a measure of emotional intelligence: The case for ability scales. In R., Bar-On y JDA, Parker (Ed.), The handbook of emotional intelligence: Theory, development, assessment, and application at home, school, and in the workplace, (320342). Joseey-Bass.

Miles, M. B. \& Huberman, A. M. (1994). Qualitative data analysis: An expanded sourcebook (2 $\left.{ }^{\mathrm{a}} \mathrm{ed}.\right)$. Sage.

Montoro, J. \& Pinazo, S. (2005). Evaluating Social Integration and Psychological Outcomes for Older Adults Enrolled at a University Intergenerational Program. Journal of Intergenerational Relationships, 3(3), 65-81. DOI: 10.1300/J194v03n03_05

Moreno, P., Martínez, S. y Escarbajal, A. (2018). El impacto educativo de los programas intergeneracionales: un estudio desde la escuela y las diferentes instituciones sociales implicadas. Revista Iberoamericana de Educación, 77(2), 31-54. DOI: 10.35362/rie7723158

Muñoz, J. M. (2007). La pedagogía de los espacios como discurso de la educación ambiental. Bordón. Revista de Pedagogía, 59(4), 641-657.

Navarro, A. B., Serrate, S., Muñoz, J. M. y Díaz-Orueta, U. (2018). Relationship Between Personality Traits, Generativity, and Life Satisfaction in Individuals Attending University Programs for Seniors. International Journal of Aging and Human Development, 87(2), 184-200. DOI: 10.1177/0091415017740678

Navarro, B., Latorre, J. M. y Ros, L. (2009). Inteligencia Emocional Autoinformada en la vejez, un estudio comparado con el TMMS-24. En Fernández-Berrocal (Ed.), Avances en el estudio de la Inteligencia Emocional, (pp. 331-335). Fundación Marcelino Botín.

Ortega, P. (2021). El valor de la experiencia del alumno como contenido educativo. Teoría de la Educación. Revista Interuniversitaria, 33(1), 89-110. DOI: 10.14201/teri.23615

Ortiz-Colón, A. M. (2015). Los Programas Universitarios de Personas Mayores y el Envejecimiento Activo. Formación Universitaria, 8(4), 55-62.

Pacheco, P., Villagrán, S. y Guzmán, C. (2015). Studio del campo emocional en el aula y simulación de su evolución durante un proceso de enseñanza-aprendizaje para cursos de ciencias. Estudios Pedagógicos, XLI(1), 199-217. DOI: 10.4067/S0718-07052015000100012

Pena, M., Extremera, N. y Rey, L. (2016). Las competencias emocionales: material escolar indispensable en la mochila de la vida. Padres y maestros, 368, 6-10. DOI: 10.14422/pym.i368.y2016.001

Pérez, G. (2012). Envejecimiento Activo y Solidaridad Intergeneracional. Claves para un Envejecimiento Activo. UNED.

Pérez-Escoda, N., Bisquerra, R., Filella, G. y Soldevila, A. (2010). Construcción del Cuestionario de Desarrollo Emocional de Adultos (QDE-A). REOP, 21(2), 367-379. DOI: 10.5944/reop.vol.21. num.2.2010.11539

Pérez-Fuentes, M. C., Gárquez, J. J., Molero, M. M., Martínez, A., Barragán, A. B. y Simón, M. M. (2016). Inteligencia emocional y salud en el envejecimiento: beneficios del programa PECI-PM. Actualidades en Psicología, 30(121), 11-23. DOI: 10.15517/ap.v30i121.24048 
Pérez-Fuentes, M. C., Molero, M. M., Gárquez, J. J. y Soler, F. (2014). Estimulación de la Inteligencia Emocional en mayores: El programa PECI-PM. European Journal of Investigation Health, Psychology and Education, 4(3), 329-339. DOI: 10.30552/ejihpe.v4i3.84

Pérez-González, J. C. (2012). Revisión del aprendizaje social y emocional en el mundo. En R. Bisquerra, ¿Cómo educar las emociones? La inteligencia emocional en la infancia y la adolescencia, (pp. 56-69). Hospital Sant Joan de Déu.

Pérez-González, J. C., Cejudo, J. y Benito Moreno, S. (2015). Educación emocional en la teoría y en la práctica. Síntesis.

Petrides, K. V., Frederickson, N. \& Furnham, A. (2004). The role of trait emotional intelligence in academic performance and deviant behavior at school. Personality and Individual Differences, 36, 277-293. DOI: 10.1016/S0191-8869(03)00084-9

Plaza, M., Requena, C., Rosario, I. y López, V. (2015). La relación entre el tiempo no estructurado, el ocio y las funciones cognitivas en personas mayores. European Journal of Education and Psychology, 8(2), 60-67.

Purdie, N. \& Boulton-lewis, G. (2003). The learning needs of older adults. Educational Gerontology, 29, 129-149. DOI: 10.1016/j.ejeps.2015.05.001

Rodríguez-Gómez, G., Gil-Flores, J. y García-Jiménez, E. (1996). Metodología de la investigación cualitativa. Aljibe.

Romero, C. y Pereira, C. (2011). El enfoque positivo de la educación: aportes al desarrollo humano. Teoría de la Educación. Revista Interuniversitaria, 23(2), 69-89. DOI: 10.14201/8646

Saarni, C. (2000). Emotional competence. A Developmental Perspective. En R. Bar-On, y J. Parker, J. (Eds.), The Handbook of Emotional Intelligence. Theory, Development, Assessment, and Application at Home, School, and in the Workplace. Josey-Bass.

Saavedra, E. y Reynaldos, C. (2006). Caracterización cognitiva y emocional de los estudiantes de la Universidad Católica de Maule: años 199-2001-2003. Estudios Pedagógicos, XXXII(2), 87-102. DOI: $10.4067 / \mathrm{S} 0718-07052006000200005$

Schulze, R. \& Roberts, R. (2005). Emotional intelligence. An international handbook. Hogrefe y Huber.

Schutte, N. S., Malouff, J. M. \& Thorsteinsson, E. B. (2013). Increasing Emotional Intelligence through training: status and future directions. The International Journal of Emotional Education, 5(1), 56-72.

Serrate, S., Torrijos, P. \& Navarro, A. B. (2018). Self-perception of emotional development in individuals attending university programmes for seniors: training needs and answers. Studies and Continuing Education, 41, 944-1100. DOI: 10.1080/0158037X.2018.1481379.

Sliter, M., Chen, Y. W., Withrow, S. \& Sliter, K. (2013). Older and (emotionally) smarter? Emotional intelligence as a mediator in the relationship between age and emotional labor strategies in service employees. Experimental Aging Research, 39(4), 466-479. DOI: 10.1080/0361073X.2013.808105.

Soldevilla, A., Ribes, R., Filella, G. y Aguilló, M. J. (2010). Objetivos y contenidos de un programa de educación emocional para personas mayores. Emocionát. Revista Iberoamericana de Educación, 37(5), 1-12.

Tójar, J. C. (2006). Investigación cualitativa. Comprender y actuar. La Muralla.

Torrijos, P. (2016). Desarrollo y evaluación de competencias emocionales para profesores mediante una intervención por programas. Tesis doctoral inédita. Universidad de Salamanca.

Torrijos, P. y Pérez-Escoda, N. (2014). Pensamiento constructivo y desarrollo emocional en estudiantes universitarios. En B. Barredo, R. Bisquerra, A. Blanco, N. Aguilar, A. Giner, N. Pérez, y A. Tey., I Congrés Internacional d'Educació Emocional. X Jornades d'Educació Emocional. Psicologia positiva i benestar (pp. 220-234). ISBN: 978-84-697-1225-2.

Torrijos, P. y Serrate, S. (2017). Educación emocional. Una experiencia intergeneracional. Universidad de Salamanca, Ayuntamiento de Salamanca y Universidad Pontificia de Salamanca. 
Estudios Pedagógicos XLVII N 3: 273-290, 2021

BENEFICIOS DE LOS PROGRAMAS INTERGENERACIONALES EN EL DESARROLLO DE COMPETENCIAS

EMOCIONALES

Ugarriza, N. (2001). La evaluación de la inteligencia emocional a través del inventario de BarOn (ICE) en una muestra de Lima Metropolitana. Persona, 4, 129-160.

Urrea, A., Rodríguez, S., Sala, J. \& Zárate, E. (2021). Instrumentos psicoeducativos para la intervención en Pedagogía Social: validación criterial del test situacional desarrollo de competencias socioemocionales de jóvenes (DCSE-J). Pedagogía Social. Revista Interuniversitaria, 37, 5568. DOI: 0.7179/PSRI_2021.37.03

Veloso, C., Cuadra, A., Antezana, I., Avendaño, R. y Fuentes, L. (2013). Relación entre Inteligencia Emocional, Satisfacción Vital, Felicidad Subjetiva y Resiliencia en funcionarios de Educación Especial. Estudios Pedagógicos, XXXIX(2), 355-366. DOI: 10.4067/S0718-07052013000200022

Vilaplana, C. (2010). Relación entre los Programas Universitarios para Mayores, la satisfacción durante la jubilación y la calidad de vida. Revista de Investigación Educativa, 28, 195-216. (2016). Influencia de los Programas Universitarios para Mayores sobre la mejora del rendimiento cognitivo. Ariadna: Cultura, educación y tecnología, I(3), 71-77.

Wight, R.G. Aneshensel, C. S. \& Seeman, T. E. (2002). Educational attainment, continued learning experience, and cognitive function among older men. Journal of Aging and Health, 14(2), 211236. DOI: $10.1177 / 089826430201400203$

Zabalza, M. A. (2004). Diarios de clase. Un instrumento de investigación y desarrollo profesional. Narcea.

Zeidner, M., Matthews, G. \& Roberts, R. (2009). What we know about emotional intelligence: How it affects learning, work, relationships, and our mental health. MIT Press. 